\title{
Pharmacokinetics of alkylresorcinol metabolites in human urine
}

\author{
Päivi P. Söderholm ${ }^{1,2,3 *}$, Johan E. Lundin ${ }^{4}$, Anja H. Koskela ${ }^{5}$, Matti J. Tikkanen ${ }^{1,3}$ and \\ Herman C. Adlercreutz ${ }^{1,5}$ \\ ${ }^{1}$ Folkhälsan Research Centre, Helsinki, Finland \\ ${ }^{2}$ Institute of Clinical Medicine, University of Helsinki, Finland \\ ${ }^{3}$ Department of Medicine, Division of Cardiology, Helsinki University Central Hospital, Finland \\ ${ }^{4}$ Institute for Molecular Medicine Finland, Helsinki, Finland \\ ${ }^{5}$ Division of Clinical Chemistry, University of Helsinki, Finland
}

(Received 14 October 2010 - Revised 17 February 2011 - Accepted 17 February 2011 - First published online 12 April 2011)

\section{Abstract}

Wholegrain cereals are reported to promote beneficial health effects. Wholegrain wheat and rye are almost exclusive sources of alkylresorcinols, and intact alkylresorcinols together with their plasma and urinary metabolites, 3-(3,5-dihydroxyphenyl)-1-propanoic acid (DHPPA) and 3,5-dihydroxybenzoic acid (DHBA), have been proposed as biomarkers of the intake of these foods in humans. The pharmacokinetics of alkylresorcinols and their metabolites in plasma have been determined but not that of the urinary metabolites. We aimed to characterise the urinary pharmacokinetics of alkylresorcinol metabolites in humans to evaluate their potential as biomarkers of wholegrain wheat and rye. A group of fifteen volunteers followed a low-alkylresorcinol diet for $2 \mathrm{~d}$ before ingesting a single dose of rye bread, containing $100 \mathrm{mg}$ alkylresorcinols. Urine was collected between baseline $(0 \mathrm{~h})$ and $25 \mathrm{~h}$ after administration. Thereafter alkylresorcinol metabolites were quantified by HPLC with coulometric electrode array detection. Maximum excretion rates were observed at 5-6h for both metabolites, DHPPA being predominant over DHBA and also possessing a greater area under the curve $\mathrm{e}_{0-25} \mathrm{~h}$. Total urinary recovery between 0 and $25 \mathrm{~h}$ yielded $43 \%$ of ingested alkylresorcinols, and at $25 \mathrm{~h}$ significant amounts of metabolites were still retained in the body, suggesting that even a spot urine sample may be sufficient to indicate whether or not wholegrain wheat or rye is a daily dietary component. These results support the use of urinary DHPPA and DHBA as biomarkers of wholegrain wheat and rye and enable new potential for studying the association between wholegrain intake and diseases, even in the absence of dietary data.

Key words: Rye fibre: Pharmacokinetics: Alkylresorcinol metabolites: Biomarkers

Increased consumption of wholegrain foods has been associated with reduced risk of several chronic diseases such as CVD, type 2 diabetes and certain cancers ${ }^{(1-7)}$. Beneficial health effects are suggested to originate from several constituents of whole grains, including fibre, fermentable carbohydrates, antioxidants, minerals and phytochemicals ${ }^{(8)}$. Among these phytochemicals, alkylresorcinols are almost exclusively found in rye and wheat, being located in the intermediate layer, between the testa and pericarp ${ }^{(9)}$ and therefore they have been suggested to serve as objective measures for the intake (biomarkers) of these two cereals ${ }^{(10)}$. Biomarkers enable the evaluation of intake of certain foods even if there are problems concerning the identification and documentation of these food items ${ }^{(11-14)}$; alkylresorcinols or their metabolites may fill this role for wholegrain wheat and rye. According to Ross et al. ${ }^{(15)}$ Finns have the highest alkylresorcinol intake among several populations studied and we have previously shown that in Finnish women, the urinary alkylresorcinol metabolites reflect cereal fibre (wheat + rye) intake ${ }^{(16)}$. Alkylresorcinols (1,3-dihydroxy-5-alkylbenzene homologues) are members of a large group of phenolic lipids, which, in rye and wheat, occur mainly bound to saturated oddnumbered hydrocarbon side-chains ${ }^{(17,18)}$. In addition to having potential as biomarkers, they eventually possess a large variety of bioactivities in vitro, such as antimicrobial, antioxidative and antimutagenic activities, as well as interaction with some proteins ${ }^{(17,18)}$. Alkylresorcinols are reported to be stable during food processing and baking ${ }^{(10)}$, and after extremely high intake, minute amounts of intact, albeit conjugated, alkylresorcinols have been identified in urine ${ }^{(19)}$. However, typically after ingestion, these lipophilic compounds are converted to water-soluble metabolites in excretable form,

Abbreviations: AUC, area under the curve; DHBA, 3,5-dihydroxybenzoic acid; DHPPA, 3-(3,5-dihydroxyphenyl)-1-propanoic acid; ER max , maximum excretion rate; $\mathrm{t}_{1 / 2}$, half-life.

*Corresponding author: Päivi P. Söderholm, fax +35891912 5452, email paivi.soderholm@helsinki.fi 
similar to tocopherols (vitamin $\mathrm{E})^{(17,19,20)}$. The two urinary alkyresorcinol metabolites 3-(3,5-dihydroxyphenyl)-1-propanoic acid (DHPPA) and 3,5-dihydroxybenzoic acid (DHBA) were first described by Ross et al. ${ }^{(19)}$; these too have been proposed to serve as biomarkers for wholegrain rye and wheat intake ${ }^{(21,22)}$ like their intact precursors, plasma alkylresorcinols ${ }^{(23,24)}$, and the more recently determined plasma metabolites DHPPA and $\mathrm{DHBA}^{(20,25)}$. In order to evaluate the suitability of these compounds to serve as biomarkers, pharmacokinetic data are helpful. The kinetics of plasma alkylresorcinols $^{(26)}$ and plasma DHBA and DHPPA ${ }^{(20)}$ have been described, but to our knowledge the kinetics of urinary DHBA and DHPPA have not been determined previously. In our present study we aimed to determine the pharmacokinetics of urinary DHBA and DHPPA in healthy subjects after a single dose of rye bread.

\section{Subjects and methods}

\section{Chemicals and materials}

Acetonitrile and methanol were obtained from Rathburn Chemicals Ltd (Walkenburg, Scotland, UK). Ortho-phosphoric acid was purchased from Riedel-de Haën (Seelze, Germany). Acetic acid, potassium dihydrogenphosphate and sodium acetate were purchased from Merck GmbH (Darmstadt, Germany). $\beta$-Glucuronidase was obtained from Roche Diagnostics $\mathrm{GmbH}$ (Mannheim, Germany), and sulfatase and syringic acid from Sigma-Aldrich Co. (St Louis, MO, USA). DHBA was obtained from Aldrich (Steinheim, Germany) and DHPPA from IsoSep AB (Tollinge, Sweden). The sourdough-processed, yeastleavened rye bread, consisting of (by dry weight) wholegrain rye $(68.3 \%)$, added rye bran $(8.3 \%)$, refined wheat flour (20\%), barley and rye malt extract, mineral salt, magnesium sulfate, potassium chloride and yeast (altogether 3.3\%), was specially baked for our studies by Fazer Bakeries (Lahti, Finland). For nutrient content, see Table 1.

\section{Subjects and study design}

The basic characteristics of the study participants are shown in Table 2. A total of fifteen healthy volunteers participated in the study after completing an eligibility questionnaire and attending a screening blood test.

The present study was conducted according to the guidelines laid down in the Declaration of Helsinki and all

Table 1. Nutrient content of the rye bread ${ }^{\star}$ per $100 \mathrm{~g}$ fresh weight and per test dose

\begin{tabular}{lcc}
\hline Nutrient & Per 100 g & Per test dose (198 g) \\
\hline Energy (kJ) & 800 & 1584 \\
Protein (g) & 6.5 & 12.9 \\
Carbohydrates (g) & 36 & 71.3 \\
Fat (g) & 1.3 & 2.6 \\
Fibre (g) & 9.4 & 18.6 \\
Alkylresorcinols (mg) & 50.5 & 100 \\
Na (g) & 0.3 & 0.6 \\
\hline
\end{tabular}

*Fazer Bakeries, Lahti, Finland.
Table 2. Basic characteristics of the participants (Mean values and standard deviations)

\begin{tabular}{lcrr}
\hline Variable & Mean & SD \\
\hline$n$ & & 15 & \\
$\quad$ Females & & 8 & \\
$\quad$ Males & & 7 & \\
Age (years) & 24.3 & & 5.3 \\
Weight $(\mathrm{kg})$ & 68.3 & & 10.9 \\
BMl $\left(\mathrm{kg} / \mathrm{m}^{2}\right)$ & 22.1 & & $2 \cdot 0$ \\
\hline
\end{tabular}

procedures involving human subjects were approved by the Ethics Committee at the Helsinki University Central Hospital, Helsinki, Finland. Written informed consent was obtained from all subjects.

The participants were asked to avoid wholegrain rye and wheat products for $2 \mathrm{~d}$ before the study to minimise alkylresorcinol intake. After overnight fasting, the baseline urine sample was collected. Thereafter the participants ingested a single dose (198g) of rye bread (Table 1) containing $100 \mathrm{mg}$ (258 $\mu \mathrm{mol}$ ) alkylresorcinols together with $21 \mathrm{~g}$ butter. Fat was considered an important supplement to ensure the absorption of the highly lipophilic alkylresorcinols. However, vegetable margarine was not served for the concern of longchain TAG potentially retarding the intestinal absorption of alkylresorcinols similarly to that of vitamin $\mathrm{E}^{(27)}$. The participants were allowed $25 \mathrm{~min}$ for the consumption of the rye bread and butter. Standardised meals and drinks not containing wholegrain cereals were served throughout the study (ham and potato casserole, chicken soup, refined wheat bread, cucumber, lettuce, yogurt, coffee, tea, milk, water), and the participants were advised to avoid consuming any other foods.

After the ingestion of rye bread and butter, all urine was collected for the following $25 \mathrm{~h}$ at twelve time points 3, 4, 5, $6,7,8,10,12,14$ and $16 \mathrm{~h}$. During the night-time (between 16 and $24 \mathrm{~h}$ ) participants collected the urine in a container. The collection was completed on the following morning at $25 \mathrm{~h}$.

\section{Analytical methods}

Rye bread was analysed for alkylresorcinols using the method of Ross et $a l^{(10)}$. The volume of the urine sample was measured and $10 \mathrm{ml}$ of each sample was stored at $-20^{\circ} \mathrm{C}$ until the analysis of DHBA and DHPPA by the method of Koskela et al. ${ }^{(28)}$. In detail, the procedure was as follows: a $25 \mu \mathrm{l}$ urine sample was hydrolysed overnight at $37^{\circ} \mathrm{C}$ with an equal volume $(25 \mu \mathrm{l})$ of hydrolysis solution $(0 \cdot 1$ mm-sodium acetate buffer ( $\mathrm{pH} 5), \quad \beta$-glucuronidase $(0 \cdot 2$ $\mathrm{U} / \mathrm{ml}$ ) and sulfatase $(2 \mathrm{U} / \mathrm{ml})$ ). After incubation, 318.5 $\mathrm{ng}$ of the internal standard syringic acid was added in $50 \mu \mathrm{l}$ methanol and $650 \mu \mathrm{l}$ HPLC mobile phase (20\% phase B-80\% phase A; for compositions, see below) and $10 \mu \mathrm{l}$ was analysed with HPLC (ESA Biosciences, Inc., Chelmsford, MA, USA) equipped with a model 540 autosampler, two model 580 solvent pumps, and a model 5600 coulometric electrode array detector (CEAD) with eight electrode pairs. The analytes were 
separated using mobile phases consisting of 50 mm-phosphate buffer ( $\mathrm{pH}$ 2.3)-methanol (90:10, v/v) (phase A) and 50 mm-phosphate buffer ( $\mathrm{pH}$ 2.3)-methanol-acetonitrile (40:40:20, by vol.) (phase B), with a 25 -min linear gradient from 0 to $100 \%$ phase $\mathrm{B}$, thereafter $12 \mathrm{~min} 100 \%$ phase $\mathrm{B}$, and the column was re-equilibrated with $0 \% \mathrm{~B}$ for $15 \mathrm{~min}$, total flow was $0.3 \mathrm{ml} / \mathrm{min}$. The analytical column was an Inertsil ODS-3 (GL Sciences Inc., Tokyo, Japan), $3 \times 150 \mathrm{~mm}$ with particle size $3 \mu \mathrm{m}$, connected to a Quick Release RP-18 (Upchurch Scientific Inc., Oak Harbor, WA, USA) $3 \times 10 \mathrm{~mm}$ guard column. DHBA was quantified at $670 \mathrm{mV}$, DHPPA at $570 \mathrm{mV}$ and syringic acid at $380 \mathrm{mV}$.

\section{Pharmacokinetic analysis}

The highest excretion rate of DHBA and DHPPA between baseline $(0 \mathrm{~h})$ and $25 \mathrm{~h}$ after the rye bread intake is defined as the maximum excretion rate $\left(\mathrm{ER}_{\max } ; \mu \mathrm{mol} / \mathrm{h}\right)$, with $\mathrm{t}_{\max }$ being the time at which $\mathrm{ER}_{\max }$ is reached. The half-life $\left(\mathrm{t}_{1 / 2}\right)$ is defined as the time at which the urinary excretion rate has decreased to half of $\mathrm{ER}_{\max }$. The area under the curve (AUC) as well as other pharmacokinetic parameters were calculated using STATA software (version 10.0; StataCorp, College Station, TX, USA).

\section{Statistical analysis}

Data on DHPPA and DHBA excretion rates are presented as means and standard deviations. The pharmacokinetic parameters $\mathrm{ER}_{\max }, \mathrm{t}_{\max }, \mathrm{t}_{1 / 2}$ and AUC for DHPPA and DHBA were statistically analysed using unpaired $t$ tests (STATA 10.0; StataCorp LP, College Station, TX, USA) to clarify whether there are significant differences between these two metabolites. The groups of females and males were compared using unpaired $t$ tests to determine potential differences in the pharmacokinetic parameters between the sexes. $P<0.05$ was considered significant.

\section{Results}

All the participants consumed the test dose within the given $25 \mathrm{~min}$; thus all had an intake of $100 \mathrm{mg}$ rye alkylresorcinols, of which the relative homologue composition was $\mathrm{C} 15: 0$ (1\%), C17:0 (27\%), C19:0 (28\%), C21:0 (22\%), C23:0 (12\%) and C25:0 (10\%).

The mean urine DHPPA and DHBA excretion rates at each time point for the whole group ( $n$ 15) are presented in Fig. 1, and the pharmacokinetic data in Table 3. Maximum concentration $(\mu \mathrm{mol} / \mathrm{ml}$ urine) values are not used in the present study because of vast variation in urine volumes, both between individuals and also collection time points. Instead, the excretion rates of DHBA and DHPPA for each $1 \mathrm{~h}$ are calculated from the samples by first correcting for the volume of the sample and then dividing by the amount of time (in h) that had passed since previous collection time point. Therefore the use of creatinine as a corrector for urinary volume was not needed. The mean baseline $(0 \mathrm{~h})$ urinary excretion rates for DHPPA and DHBA were low; 0.737

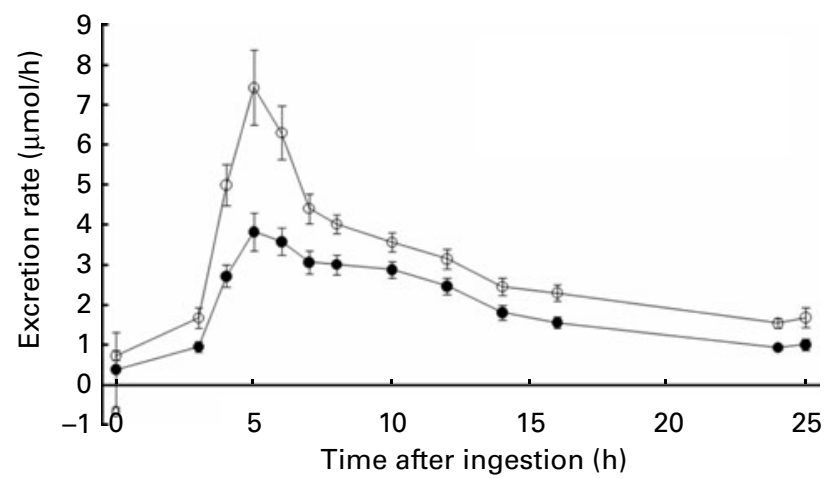

Fig. 1. Urinary pharmacokinetics of alkylresorcinol metabolites between baseline $(0 \mathrm{~h})$ and $25 \mathrm{~h}$ after consumption of a single dose of rye bread containing $258 \mu \mathrm{mol}$ alkylresorcinols $(n 15)$. Values are means, with standard errors of the mean represented by vertical bars. The time point of $24 \mathrm{~h}$ represents the overnight urine collection between 16 and $24 \mathrm{~h}$; other time points represent spot urine samples. (-O-), 3-(3,5-Dihydroxyphenyl)-1-propanoic acid; (-๑-), 3,5-dihydroxybenzoic acid.

(SD 0.46$)$ and $0.386(\mathrm{SD} 0.36) \mu \mathrm{mol} / \mathrm{h}$, respectively, indicating that participants had avoided alkylresorcinol-containing foods before the study. At $3 \mathrm{~h}$ after consuming the bread dose, urinary excretion rates rose rapidly and every participant reached $\mathrm{ER}_{\max }$ fairly simultaneously for both metabolites (data not shown); thirteen of the fifteen participants reached $\mathrm{ER}_{\max }$ for DHPPA and DHBA between 5 and $6 \mathrm{~h}$, while one reached $\mathrm{ER}_{\max }$ at $3 \mathrm{~h}$ and another at $10 \mathrm{~h}$. DHPPA was the major metabolite in all participants, reaching higher $\mathrm{ER}_{\max }$ and AUC than DHBA $(P<0 \cdot 0001$ for both). Excretion rates declined gradually after $\mathrm{ER}_{\max }$ and the mean $\mathrm{t}_{1 / 2}$ for DHPPA and DHBA was reached at 11.9 and $9.9 \mathrm{~h}$, respectively $(P=0.142)(n 12)$. In three participants there were multiple peaks and hence the $t_{1 / 2}$ could not be determined for them (data not shown). The final $(25 \mathrm{~h})$ urinary excretion rates for DHPPA and DHBA (1.68 (SD 0.96) and 1.01 (SD 0.57) $\mu \mathrm{mol} / \mathrm{h}$, respectively) were significantly different from baseline ( $P=0.001$ for both). The mean total urinary recovery of the two metabolites at $25 \mathrm{~h}$ was $43.4 \%$ calculated from the ingested alkylresorcinols. There were no significant differences between females and males in any of the pharmacokinetic parameters $(P>0 \cdot 168$ for all; Table 3).

\section{Discussion}

The present study reports the pharmacokinetics of urinary DHPPA and DHBA, which to our knowledge have not been determined before. The present study strengthens the suggestions that these compounds are useful biomarkers for the intake of wholegrain wheat and rye ${ }^{(21,22)}$. Wholegrain cereals are of increasing interest for their health-promoting effects and the present data might be helpful when analysing existing samples from previous trials or databanks without dietary documentation. The wash-out period needed before the present study as well as the time points for sampling were determined after conducting a pilot study. According to that data we were expecting a very low rate of alkylresorcinol metabolites after $25 \mathrm{~h}$ and therefore a $25 \mathrm{~h}$ collection period 
Table 3. Pharmacokinetic parameters of alkylresorcinol metabolites in human urine collected between baseline $(0 \mathrm{~h})$ and $25 \mathrm{~h}$ after administration of a single dose of rye bread containing $258 \mu \mathrm{mol}$ alkylresorcinols

(Mean values and standard deviations)

\begin{tabular}{|c|c|c|c|c|c|c|c|c|c|c|c|c|}
\hline \multirow[b]{2}{*}{ Metabolite } & \multicolumn{2}{|c|}{$\begin{array}{c}\text { Baseline } \\
\text { concentration } \\
\quad(\mu \mathrm{mol})\end{array}$} & \multicolumn{2}{|c|}{$\begin{array}{c}\mathrm{ER}_{\max } \\
(\mu \mathrm{mol} / \mathrm{h})\end{array}$} & \multicolumn{2}{|c|}{$\mathrm{t}_{\max }(\mathrm{h})$} & \multicolumn{2}{|c|}{ AUC $(\mu \mathrm{mol} / 25 \mathrm{~h})$} & \multicolumn{2}{|c|}{$t_{1 / 2}(h)^{*}$} & \multicolumn{2}{|c|}{ Recovery (\%) } \\
\hline & Mean & SD & Mean & SD & Mean & SD & Mean & SD & Mean & SD & Mean & SD \\
\hline \multicolumn{13}{|l|}{ DHPPA } \\
\hline All $(n 15)$ & 0.74 & 0.46 & $8 \cdot 15$ & 3.51 & 5.40 & 0.63 & $67 \cdot 26$ & $16 \cdot 98$ & 11.89 & $5 \cdot 30$ & $26 \cdot 11$ & 6.59 \\
\hline Females $(n 8)$ & 0.69 & 0.46 & $8 \cdot 76$ & 4.02 & $5 \cdot 38$ & 0.52 & 66.63 & $15 \cdot 72$ & 13.44 & $6 \cdot 67$ & $25 \cdot 87$ & $6 \cdot 10$ \\
\hline Males $(n 7)$ & 0.79 & 0.50 & 7.46 & 3.00 & 5.43 & 0.79 & 67.98 & 19.57 & $10 \cdot 35$ & 3.41 & $26 \cdot 39$ & $7 \cdot 60$ \\
\hline \multicolumn{13}{|l|}{ DHBA } \\
\hline All $(n 15)$ & 0.39 & 0.36 & 4.46 & 1.72 & $6 \cdot 0$ & 1.73 & 44.65 & 13.69 & 9.93 & $2 \cdot 61$ & $17 \cdot 33$ & $5 \cdot 31$ \\
\hline Females ( $n$ 8) & 0.38 & 0.39 & 4.60 & $2 \cdot 04$ & $6 \cdot 0$ & 1.69 & 43.49 & $14 \cdot 35$ & 9.93 & $2 \cdot 10$ & $16 \cdot 88$ & 5.57 \\
\hline Males $(n 7)$ & 0.40 & 0.36 & $4 \cdot 31$ & 1.43 & $6 \cdot 0$ & 1.91 & 45.98 & 13.91 & 9.93 & 3.25 & 17.85 & $5 \cdot 40$ \\
\hline
\end{tabular}

$\mathrm{ER}_{\max }$, maximum excretion rate; $\mathrm{t}_{\max }$, time to reach maximum excretion rate; AUC, area under the curve; $\mathrm{t}_{1 / 2}$, half-life; DHPPA, 3-(3,5-dihydroxyphenyl)-1-propanoic acid; DHBA, 3,5-dihydroxybenzoic acid.

${ }^{*}$ For $\mathrm{t}_{1 / 2}$ : all subjects, $n 12$; females, $n 6$; males, $n 6$.

was regarded sufficient, and a $2 \mathrm{~d}$ wash-out period before the study was considered appropriate. In the present study we observed very low baseline values, indicating that this washout period was successful. After consumption of rye bread the excretion rate for DHPPA and DHBA increased rapidly, reaching maximum values between 5 and $6 \mathrm{~h}$. Conversely, the decrease in the excretion rate was quite slow for both metabolites. The lowest excretion rates were detected during the overnight collection (16-24h), indicating a slight slowdown of alkylresorcinol metabolism during the sleeping hours. At $25 \mathrm{~h}$ the final excretion rates for both metabolites were still more than double compared with baseline, but, on the other hand, the rates during the last $1 \mathrm{~h}$ accounted only for about $2.5 \%$ of the $\mathrm{AUC}_{0-25 \mathrm{~h}}$. Despite this, the total recovery of the two alkylresorcinol metabolites in urine at $25 \mathrm{~h}$ accounted only for $43 \%$ of the ingested alkylresorcinols, being similar to values reported by Landberg et al. ${ }^{(21)}$. The low urinary recovery is in concordance with recent findings. Firstly, Ross et al. ${ }^{(23)}$ described the absorption of alkylresorcinols to be about $60 \%$ and in another study on pigs ${ }^{(29)}$ they suggested a decrease in absorption with higher levels of intake. Furthermore, Landberg et al. ${ }^{(21)}$ reported the urinary recovery of alkylresorcinols to decrease with increasing dose from $90 \%$ to approximately $45 \%$. Thus, one could speculate that a lower dose of alkylresorcinols with a higher rate of absorption as well as urinary recovery would probably give quite similar pharmacokinetic curves as observed in the present study. Second, it is possible that from the absorbed amount of alkylresorcinols, a proportion may still be stored or delayed in the body. This is supported by our recently reported plasma pharmacokinetic data of DHPPA and DHBA which indicated that at the time point of $25 \mathrm{~h}$ after consumption of the same rye bread, significant amounts of the alkylresorcinol metabolites remained in the circulation ${ }^{(20)}$. Besides alkylresorcinols, also alkenylresorcinols with unsaturated hydrocarbon side-chains are known to exist in wholegrain wheat and rye. For the rye bread used in the present study, alkenylresorcinols accounted for about $20 \%$ of the total amount of alkylresorcinols (data not shown). The metabolism of alkenylresorcinols has not been studied yet but if they too are precursors of DHBA and DHPPA, the urinary recovery of these metabolites as calculated above is overestimated.

Nevertheless, the present pharmacokinetic data suggest that even a single spot urine sample, collected after overnight fasting, potentially can act as a qualitative or semi-quantitative biomarker for the intake of wholegrain wheat or rye during the previous day and indeed distinguish between the consumers and non-consumers of wholegrain wheat and rye. However, to clarify this, a larger set of subjects would be needed for the comparison of the $24 \mathrm{~h}$ urine collections with spot urine samples.

The urinary pharmacokinetics of alkylresorcinol metabolites appear to closely reflect those reported for plasma ${ }^{(20)}$. However, in contrast to the findings in plasma, urinary DHPPA achieved higher concentrations than DHBA, possibly explaining why the opposite ${ }^{(20)}$ was observed in plasma. The formation of DHPPA from alkylresorcinols requires fewer metabolic steps - at least one less - compared with DHBA and it may therefore be excreted more rapidly.

The relatively small number of study participants and the large variation between individuals, which might be explained by differences in intestinal microbiota and absorption as well as the extent of metabolism in the liver and enterohepatic circulation, can be considered as limitations of the present study. Further studies are needed to understand the metabolism and excretion of alkylresorcinols in human subjects.

Taken together, our data suggest that urinary DHPPA and DHBA can be utilised as biomarkers for wholegrain wheat and rye intake, which is particularly helpful in the absence of dietary data. In addition, even a spot urine sample may be used to find out whether or not wholegrain wheat or rye is a daily dietary component.

\section{Acknowledgements}

We thank the participants for making the study possible, Päivi Ihamuotila, Paula Kokko, Merja Lahtinen and Päivi Ruha for skilful sample treatment and Adile Samaletdin for analysing the rye bread used in the study. The present study was supported by the Sigrid Jusélius Foundation, Helsinki, 
Finland, Samfundet Folkhälsan, Helsinki, Finland and Fazer Bakeries, Vantaa and Lahti, Finland who also provided the test bread used in the study. The authors' contributions were as follows: P. P. S., A. H. K., M. J. T. and H. C. A. designed the study; P. P. S. conducted most of the experimental work; A. H. K. carried out the laboratory analyses; J. E. L. conducted statistical and pharmacokinetic analyses; M. J. T. and H. C. A. supervised the work; P. P. S. wrote the paper; P. P. S., M. J. T. and $\mathrm{H}$. C. A. had primary responsibility for the final content. All of the authors read and approved the manuscript.

M. J. T. received a grant from Fazer Bakeries (Finland) in support of the dietary study. The other authors declare no conflicts of interest.

\section{References}

1. Schatzkin A, Park Y, Leitzmann MF, et al. (2008) Prospective study of dietary fiber, whole grain foods, and small intestinal cancer. Gastroenterology 135, 1163-1167.

2. Anderson JW (2003) Whole grains protect against atherosclerotic cardiovascular disease. Proc Nutr Soc 62, 135-142.

3. Seal CJ (2006) Whole grains and CVD risk. Proc Nutr Soc 65 , 24-34.

4. McKeown NM, Meigs JB, Liu S, et al. (2002) Whole-grain intake is favorably associated with metabolic risk factors for type 2 diabetes and cardiovascular disease in the Framingham Offspring Study. Am J Clin Nutr 76, 390-398.

5. Montonen J, Knekt P, Jarvinen R, et al. (2003) Whole-grain and fiber intake and the incidence of type 2 diabetes. $\mathrm{Am}$ J Clin Nutr 77, 622-629.

6. Murtaugh MA, Jacobs DR, Jacob B, et al. (2003) Epidemiological support for the protection of whole grains against diabetes. Proc Nutr Soc 62, 143-149.

7. Larsson SC, Giovannucci E, Bergkvist L, et al. (2005) Whole grain consumption and risk of colorectal cancer: a population-based cohort of 60000 women. Br J Cancer 92, 1803-1807.

8. Slavin J (2003) Why whole grains are protective: biological mechanisms. Proc Nutr Soc 62, 129-134.

9. Landberg R, Kamal-Eldin A, Salmenkallio-Marttila M, et al. (2008) Localization of alkylresorcinols in wheat, rye and barley kernels. J Cereal Sci 48, 401-406.

10. Ross AB, Shepherd MJ, Schupphaus M, et al. (2003) Alkylresorcinols in cereals and cereal products. I Agric Food Chem 51, 4111-4118.

11. Westerterp KR \& Goris AHC (2002) Validity of the assessment of dietary intake: problems of misreporting. Curr Opin Clin Nutr Metab Care 5, 489-493.

12. Bingham S, Luben R, Welch A, et al. (2008) Associations between dietary methods and biomarkers, and between fruits and vegetables and risk of ischaemic heart disease, in the EPIC Norfolk Cohort Study. Int J Epidemiol 37, 978-987.

13. Jenab M, Slimani N, Bictash M, et al. (2009) Biomarkers in nutritional epidemiology: applications, needs and new horizons. Hum Genet 125, 507-525.
14. Potischman N \& Freudenheim JL (2003) Biomarkers of nutritional exposure and nutritional status: an overview. J Nutr 133, 873S-874S.

15. Ross $\mathrm{AB}$, Becker W, Chen $\mathrm{Y}$, et al. (2005) Intake of alkylresorcinols from wheat and rye in the United Kingdom and Sweden. Br J Nutr 94, 496-499.

16. Aubertin-Leheudre M, Koskela A, Marjamaa A, et al. (2008) Plasma alkylresorcinols and urinary alkylresorcinol metabolites as biomarkers of cereal fiber intake in Finnish women. Cancer Epidemiol Biomarkers Prev 17, 2244-2248.

17. Ross AB, Kamal-Eldin A \& Åman P (2004) Dietary alkylresorcinols: absorption, bioactivities, and possible use as biomarkers of whole-grain wheat- and rye-rich foods. Nutr Rev 62, 81-95.

18. Kozubek A \& Tyman JHP (1999) Resorcinolic lipids, the natural non-isoprenoid phenolic amphiphiles and their biological activity. Chem Rev 99, 1-26.

19. Ross AB, Åman P \& Kamal-Eldin A (2004) Identification of cereal alkylresorcinol metabolites in human urine-potential biomarkers of wholegrain wheat and rye intake. J Chromatogr B Analyt Technol Biomed Life Sci 809, 125-130.

20. Soderholm PP, Koskela AH, Lundin JE, et al. (2009) Plasma pharmacokinetics of alkylresorcinol metabolites: new candidate biomarkers for whole-grain rye and wheat intake. Am J Clin Nutr 90, 1167-1171.

21. Landberg R, Aman P, Friberg LE, et al. (2009) Dose response of whole-grain biomarkers: alkylresorcinols in human plasma and their metabolites in urine in relation to intake. Am J Clin Nutr 89, 290-296.

22. Guyman LA, Adlercreutz H, Koskela A, et al. (2008) Urinary 3-(3,5-dihydroxyphenyl)-1-propanoic acid, an alkylresorcinol metabolite, is a potential biomarker of whole-grain intake in a U.S. population. J Nutr 138, 1957-1962.

23. Ross AB, Kamal-Eldin A, Lundin EA, et al. (2003) Cereal alkylresorcinols are absorbed by humans. J Nutr $\mathbf{1 3 3}$, 2222-2224.

24. Linko A-M, Juntunen KS, Mykkanen HM, et al. (2005) Whole-grain rye bread consumption by women correlates with plasma alkylresorcinols and increases their concentration compared with low-fiber wheat bread. J Nutr $\mathbf{1 3 5}$, $580-583$.

25. Koskela A, Samaletdin A, Aubertin-Leheudre M, et al. (2008) Quantification of alkylresorcinol metabolites in plasma by high-performance liquid chromatography with coulometric electrode array detection. J Agric Food Chem 56, 7678-7681.

26. Landberg R, Linko A-M, Kamal-Eldin A, et al. (2006) Human plasma kinetics and relative bioavailability of alkylresorcinols after intake of rye bran. J Nutr 136, 2760-2765.

27. Borel P (2003) Factors affecting intestinal absorption of highly lipophilic food microconstituents (fat-soluble vitamins, carotenoids and phytosterols). Clin Chem Lab Med 41, 979-994.

28. Koskela A, Linko-Parvinen A-M, Hiisivuori P, et al. (2007) Quantification of alkylresorcinol metabolites in urine by HPLC with coulometric electrode array detection. Clin Chem 53, 1380-1383.

29. Ross AB, Shepherd MJ, Bach Knudsen KE, et al. (2003) Absorption of dietary alkylresorcinols in ileal-cannulated pigs and rats. BrJ Nutr 90, 787-794. 